疍

Literaturwissenschaft und Sozialwissenschaften 3 


\section{Literaturwissenschaft und Sozialwissenschaften 3}

Deutsches Bürgertum und literarische Intelligenz

1750-1800

Mit Beiträgen von Ulrich Dzwonek, Hans Freier, Hans Jürgen Haferkorn, Thomas Metscher, Volker Ulrich Müller, Claus Ritterhoff, Harro Segeberg, Anthony Williams und Harro Zimmermann herausgegeben von Bernd Lutz 
ISBN 978-3-476-00275-4

ISBN 978-3-476-03025-2 (eBook)

DOI 10.1007/978-3-476-03025-2

(c) Springer-Verlag GmbH Deutschland 1974

Ursprünglich erschienen bei J. B. Metzlersche Verlagsbuchhandlung

und Carl Ernst Poeschel Verlag GmbH in Stuttgart 1974 


\section{Inhalt}

Bernd Lutz: Einleitung . . . . . . . . . . . . . . . . . IX

Anthony Williams: The ambivalences in the plays of the young Schiller about contemporary Germany . . . . . . . . . . . . . . . . 1

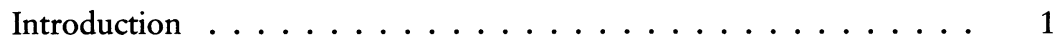

Kabale und Liebe . . . . . . . . . . . . . . . . . . . . 34

Die Räuber . . . . . . . . . . . . . . . . . . . . 49

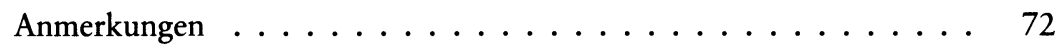

Hans J. Haferkorn: Zur Entstehung der bürgerlich-literarischen Intelligenz und des Schriftstellers im Deutschland zwischen 1750 und 1800 . . 113

Vorbemerkung . . . . . . . . . . . . . . . . 114

Einleitung . . . . . . . . . . . . . . . . . . 128

I. Freiheit und soziale Determination des Schriftstellers . . . . . . . . 138

1. Der Begriff Freiheit . . . . . . . . . . . . . . . . 138

2. Schriftsteller und Volk . . . . . . . . . . . . . . . 142

3. Grenze und Konflikt . . . . . . . . . . . . . . . . . . 157

4. Soziale Determination . . . . . . . . . . . . . . . 163

5. Sprache und Geschmack . . . . . . . . . . . . . . . 171

II. Bürgerliche Gesellschaft und literarische Kultur . . . . . . . . . . 176

1. Entwicklung der bürgerlichen Gesellschaft . . . . . . . . . 177

2. Die Lage des Bürgertums . . . . . . . . . . . . . . . 182

3. Ästhetisierung . . . . . . . . . . . . . . . . . . . . 190

III. Schriftsteller und literarischer Markt . . . . . . . . . . . . . . 195

1. Kommerzialisierung . . . . . . . . . . . . . . . . . 196

2. Geistiges Eigentum und Honorar . . . . . . . . . . . . . 203

3. Selbsthilfemaßnahmen . . . . . . . . . . . . . . 210

IV. Das Selbstverständnis des Schriftstellers . . . . . . . . . . . . . 214

1. Der ständische Dichter . . . . . . . . . . . . . . . . 215

2. Der freie Schriftsteller . . . . . . . . . . . . . 222

3. Beruf und Berufung . . . . . . . . . . . . . . . 225

4. Schriftsteller und bürgerliche Gesellschaft . . . . . . . . . . . 239

Anmerkungen . . . . . . . . . . . . . . . . 239

Ulrich Dzwonek/Claus Ritterhoff/Harro Zimmermann: "Bürgerliche Oppositionsliteratur zwischen Revolution und Reformismus«. F. G. Klopstocks Deutsche Gelehrtenrepublik und Bardendichtung als Dokumente der bürgerlichen Emanzipationsbewegung in der zweiten Hälfte des 18. Jahrhunderts . . . . . . . . . . . . . . . . . 277 Vorbemerkung . . . . . . . . . . . . . . . . . . . . 277 
I. Zur Suskriptionsleserschaft von Klopstocks Deutscher Gelehrtenrepublik .......................

II. Die Deutsche Gelehrtenrepublik als Dokument emanzipatorischer Politik und Poetik ..................

1. Radikalisierungstendenzen der politischen Debatte in den secziger und siebziger Jahren des 18. Jahrhunderts . . . . . . . . 282

2. Zur Genesis der Deutschen Gelehrtenrepublik . . . . . . . . 286

3. Der Übergang von der feudalen zur bürgerlichen Gesellschaft Zur Organisationsstruktur der Deutschen Gelehrtenrepublik . . .

4. Zum Begriff der Politik in Klopstocks Deutscher Gelehrtenrepublik

5. Zu Klopstocks Konzeption einer Poetik in der Gelehrtenrepublik .

III. Die Rezeption der Klopstockschen Bardendichtung . . . . . . . . . .

1. Das Grundproblem der wissenschaftlichen und ästhetischen Rezeption des Bardenstoffes im Deutschland des 18. Jahrhunderts

2. Zu Klopstocks Bardendichtung . . . . . . . . . . . . . . 303

3. Positionen der Kritik an Klopstocks Bardendichtung . . . . . . . . 304

Anmerkungen . . . . . . . . . . . . . . . . . 313

Hans Freier: Ästhetik und Autonomie. Ein Beitrag zur idealistischen Entfremdungskritik ................

1. Das Problem der Kunstautonomie . . . . . . . . . . . . .

2. Das Problem ästhetischer Autonomie . . . . . . . . . . . .

Anmerkungen . . . . . . . . . . . . .

Thomas Metscher: Prometheus. Zum Verhältnis von bürgerlicher Literatur und materieller Produktion . . . . . . . . . . . .

I. Der bürgerliche Autor als autonomer Produzent - „ich trete die Kelter allein" ....................

II. Himmlisches Feuer und Dampfmaschine oder Prometheus und die menschliche Arbeit ................

III. Das bürgerliche Ich als Welt-Produzent oder die Revolution in Form des Gedankens . . . . . . . . . . . . . . . . . . . . . 400

IV. Prometheus und Epimetheus - materielle und geistige Produktion im Zeitalter der bürgerlichen Gesellschaft . . . . . . . . . . . . . 410

V. Deutsche Literatur und bürgerliche Gesellschaft . . . . . . . . . . . 416

VI. Der Prometheus der Arbeiterklasse . . . . . . . . . . . . . . . . 425

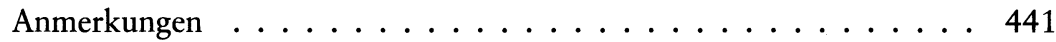

Volker Ulrich Müller: Die Empfindsamkeitskritik bei Jean Paul . . . . . 455

I. Ästhetik und Politik. Zu Problemen der Jean-Paul-Forschung . . . . 455

II. Inszenierte Naivität . . . . . . . . . . . . . . . . . . . 459

III. Kritik und Reflexion. Die Suche nach einem archimedischen Punkt . . 466

IV. Zur Genese humoristischer Reflexion: die Antinomien einer Ideologiekritik des Irrtums . . . . . . . . . . . 
V. Selbstreflexion der Kritik und die Revolution als Akt der Beliebigkeit

VI. Kritik und Revolution. Die Suche nach einem revolutionären Subjekt

VII. Verdrängte Reflexion. Heldentum und Gemeinwohl: der Rückgriff auf das römische Kostüm . . . . . . . . . . . . . . . . 485 Anmerkungen ....................... 496

Harro Segeberg: Literarischer Jakobinismus in Deutschland. Theoretische und methodische Überlegungen zur Erforschung der radikalen Spätaufklärung . . . . . . . . . . . . . . . . . . . 509 509

1. Zur Kanonisierung der Weimarer Klassik . . . . . . . . . . . . 509

2. Zur Lage der Jakobinismusforschung . . . . . . . . . . . . . 511

3. Aufklärung und Revolution - sozialgeschichtliche Vermittlungsprobleme . . . . . . . . . . . . . . 5 515

4. Idealistische Handlungstheorien . . . . . . . . . . 520

5. Das liberale und das jakobinisierte Modell der öffentlichen Meinung 524

6. Moralisches Pathos und Klasseninteresse . . . . . . . . . 530

7. Interessenspezifische Aufklärungsliteratur . . . . . . . . . . 533

8. Politische Volkspoesie ................ 540

9. Zur operativen Technik Mainzer Revolutionsliteratur . . . . . . . 546

10. Klassik und radikale Spätaufklärung . . . . . . . . . . 550

Anmerkungen ........................ 553

Namenregister ...................... 569 


\section{Einleitung}

Warum Literaturwissenschaft und Sozialwissenschaften?

Die Beschäftigung mit der deutschen Literatur speziell des 18. Jahrhunderts, der deutschen Spätaufklärung und den Konstituierungsfragen der deutschen Klassik hat neuerdings ihren guten Grund wie ihre gute Konjunktur. Ihren guten Grund darin, daß gegen die von der nationalistisch bis geistesgeschichtlich orientierten Germanistik etablierte "Klassik-Legende ", gegen die schon Franz Mehring Sturm gelaufen war, jetzt eine mehr oder weniger systematische Erforschung der deutschen Aufklärung im Rahmen des institutionell Möglichen vorangetrieben wird. Dies geschieht, um ihr - die Grenzen einer Nationalliteraturgeschichtsschreibung aufgrund ihrer gemeineuropäischen Verflechtungen sprengend - ein eigenständiges Gewicht zu verleihen, das sie nicht mehr den nivellierenden und angesichts historischer Kontinuität zu kurz greifenden epochengeschichtlichen Triaden anheimgibt.

Dazu zwangen nicht nur die zahlreichen Arbeiten, die von Literaturwissenschaftlern der DDR hierzu vorgelegt wurden - angesiedelt zwischen den Polen des klassisch-humanistischen und des demokratisch-sozialistischen Erbes und mit Nuancierungen kulturpolitisch darauf abgestimmt, bürgerliches und sozialistisches Erbe miteinander zu versöhnen -, sondern vor allen Dingen Legitimationsprobleme innerhalb der bundesrepublikanischen Literaturwissenschaft, die sich seit Mitte der sechziger Jahre verstärkt der Provokation durch die Kritische Theorie der Frankfurter Schule ausgesetzt sah. Diese Provokation mußte bei deren Rezipienten umso tiefer treffen, als die Kritische Theorie den Versuch machte, politisch-soziale Faktoren der deutschen Geschichte im Vorfeld einer historischmaterialistischen Analyse mit den Ideologemen der deutschen Geistesgeschichte zu konfrontieren und damit einen dialektischen Funktionszusammenhang herzustellen, der erklärbar machte, weshalb im Verlauf der jüngsten deutschen Geschichte Künstler und Kunstwerk zu einem autonomen Status verklärt wurden, der deren gesellschaftliche Versöhnung nur noch in der Form ästhetisch vermittelter Affirmation ermöglichte; mit anderen Worten also das Scheinhafte des Ästhetischen umschlug in die Scheinhaftigkeit von Gesellschaft.

Die solcherart decouvrierte "deutsche Reichspropaganda « lenkte den Blick der Sozialwissenschaften in weitestem Sinn, also auch der Literaturwissenschaft auf die jeweils vorgegebenen Implikationen des sozial- und ideologiegeschichtlichen Kontextes. Daß sich dabei oft ein methodisch dubioser "Deduktionalismus " breit machte, mag angesichts der Tatsache zu verschmerzen sein, daß sich philologische Neugier und Umtriebigkeit zunehmend verstärkten und am besten da beraten waren, wo sie die positivistischen Tugenden der historischen Methode zum Ausgangspunkt nahmen. Entsprach der Begriff »bürgerlich « zunächst einer vagen 
Etikettierung des subjektiv empfundenen Unbehagens an der eigenen Wissenschaftspraxis, so war man aus Gründen der Differenzierung des Begriffsbilds sehr rasch gezwungen, auf die Entstehungsphase des deutschen Bürgertums in seiner spezifisch frühliberalistischen Prägung im späten 18. Jahrhundert zurückzugreifen.

Erst von diesem historischen Bezugspunkt aus scheint es möglich, glaubwürdig die Faktoren von Ideologieproduktion, vorrangig der Literatur des 18. Jahrhúnderts als eines Sammelbeckens klassentranszendenter oder zwangsläufig klassenneutraler Intelligenz mit einem methodischen Konzept der Aufarbeitung deutscher Geschichte unter ideologiekritischen und politisch-ökonomischen Gesichtspunkten zu vereinigen.

Dies wäre, beim gewiß »oberflächlichen «Wort genommen, ein verwegenes Programm, würde nicht von vornherein deutlich gemacht werden, daß Widerspiegelungsverhältnisse nicht in Bausch und Bogen auf den literarischen Prozeß rückübertragen werden können, weil damit eine ganze Reihe für die Ideologiebildung zentrale Faktoren schlicht annulliert werden würden. Vielmehr sind Phasenverschiebungen im Verhältnis der ideellen zur materiellen Produktion, Wiederholungen und Rückgriffe insgesamt typischer für den Verlauf der deutschen Literaturgeschichte im angedeuteten Zeitraum als eine wie auch immer begründete, letzten Endes idealtypische Kongruenz von "materieller « Basis und »literarischem « Uberbau. Der Eindruck eines scheinbar stabilen Gefüges deutscher Ideologie im 19. und 20. Jahrhundert letzten Endes auch der Literatur entstand durch andere Faktoren des ideellen Prozesses, die mit der genuinen Verfassung von Literatur wenig, mit der Praxis staatsbürgerlicher Sozialisation aber umso mehr zu tun hatten. Denn während das Bürgertum im Verlauf des 19. Jahrhunderts aus gutem Grund eine technisch-naturwissenschaftliche Intelligenz hervorbrachte und sie gezielt im ökonomischen Produktionsprozeß zur Geltung brachte, suspendierte es sich gleichzeitig von den klassischen literarischen Propagatoren des bürgerlichen Fortschritts qua "Bildung ", wo es nicht unmittelbar in Opposition zu ihnen geriet, oder umgekehrt die literarische Opposition sich aus intellektuellen Ansprüchen herleitete, die das Bürgertum aufgrund seiner Fixierung auf die wirtschaftliche Prosperität nicht mehr teilen bzw. integrieren konnte. Und es sei hinzugefügt, daß erst jetzt damit begonnen wird, die Rolle der deutschen Philologie in diesem Sozialisationsprozeß als eines der Literatur selbst verqueren Vermittlers klarzulegen.

Alle diese Momente gehören zu den mittelbaren Voraussetzungen der Reihe Literaturwissenschaft und Sozialwissenschaften, die der Verlag im Jahr 1971 mit dem Band Grundlagen und Modellanalysen in der Absicht begann, die Prämissen literaturwissenschaftlich sinnvoller Fragestellungen einem sozialwissenschaftlichen Kontext zu überantworten, ohne die infolge der deutschen Sonderentwicklung, Literatur wie Gesellschaft betreffend, notwendigen Differenzierungen vorschnell einzuebnen. Wenn der Reihentitel mit seinem »und « einer gewissen 
Vorläufigkeit, die es noch nicht auf den Begriff gebracht hat, Spielraum läßt, so nicht deshalb, um den Rückzug zum Status des autonomen Kunstwerks und entsprechend indifferenter Methoden offenzuhalten, sondern um zu verstehen zu geben, daß sich allgemeine Warenproduktion und Kulturwarenproduktion, mit der es Literaturwissenschaft gemeinhin zu tun hat, hinreichend unterscheiden, um diese Divergenz bei der Analyse ideeller Produktion in Beziehung zur materiellen beizubehalten und so oberflächliche Analogiebildungen auszuschließen.

Warum deutsches Bürgertum und literarische Intelligenz zwischen 1750 und 1800 ?

Der Titel des vorliegenden Bandes sollte nicht so verstanden werden, als ginge es seinen Autoren um die Rekonstruktion einer grundsätzlichen Differenz zwischen dem deutschen Bürgertum der Zeit und der ihm "konfrontierten " literarischen Intelligenz. Ein solches Vorverständnis verbietet sich aufgrund des Gesamtverlaufs der bürgerlich-liberalistischen Emanzipationsbewegung im späten 18. und beginnenden 19. Jahrhundert, an dem die literarische Intelligenz hervorragenden Anteil hatte. Auch sollte » deutsches Bürgertum " nicht so verstanden werden, als handle es sich dabei um einen in allen Belangen konsolidierten Klassenbegriff, weil dazu im territorial und politisch zersplitterten Deutschland im Vergleich zum englischen und französischen Zentralismus alle Voraussetzungen fehlten. Dies zeigt sich am deutlichsten im Scheitern der bürgerlichen Revolutionsversuche auf linksrheinischem Gebiet. Freilich ist die Prokalamation der Mainzer Republik ein erster demokratischer Lichtblick in der Phase des Verfalls des Feudalabsolutismus, aber man darf doch nicht darüber hinwegsehen, daß sich das Konstrukt einer bürgerlichen Klassengesellschaft schon deshalb verbietet, weil dieses Bürgertum noch nicht Sachwalter des Machtapparats war und der Klassenkompromiß mit dem Feudaladel sich schon deshalb anbot, weil letzterer ökonomisch und subsidiär zumeist in bürgerlicher Hand war. Es fehlte auf der anderen Seite ein selbstbewußtes Proletariat in den Metropolen des Handels und der politischen Machtausübung, das als politisch latenter Faktor ins öffentliche Bewußtsein hätte treten können. So blieben in Deutschland plebeijisch-jakobinisch-sansculottische Gedanken, die sich auf die Erschütterung der Legitimität des Privateigentums konzentrierten, als Sache der sozial Deklassierten und politisch Unmündigen dem freien Kommerz der Geister überlassen.

Freilich muß auch gesagt werden, daß trotz des Übergewichts, das sich der girondistische Bourgeois nach dem Thermidor mit Entschiedenheit verschafft - seine politische Emanzipation als Entfaltung seiner Handelsfreiheit betreibend -, sich bereits jene gesellschaftlichen Antagonismen abzuzeichnen beginnen, die sich in den bürgerlichen und sozialistischen Kampfpositionen, aber auch ihrer 
Bündnispolitik im Verlauf der französischen wie deutschen Geschichte des 19. Jahrhunderts voll entfalten sollten. Dies wird nicht hervorgehoben, um erneut die literarischen Parteigänger insbesondere des deutschen Bürgertums einer letzten Endes kulturkonservativen Haltung zu bezichtigen, vielmehr sollte von der deutschen Intelligenz in ihrer spezifisch literarischen Ausprägung kein allzu einhelliges Bild entstehen*. Wenn sie sich auch insgesamt aus ihrer ständisch-feudalistischen Fixierung löst und gleichsam als vierte, rationale Gewalt ins öffentliche Bewußtsein einschreibt, so gibt es doch handfeste Unterschiede, mit denen diese Intelligenz, den Blick auf die Französische Revolution gerichtet, in Deutschland mit politischen Sachen ernst macht. Der leidenschaftlichen Parteinahme für die Gleichheits- und Souveränitätsideale aller Bürger selbst unter der Bedingung revolutionärer Gewalt mag für deutsche Verhältnisse die literarische, letzten Endes aber auch aktionistische Konsequenz entsprechen, mit der versucht wurde, in die bestehenden Machtstrukturen einzugreifen und ein eigenständiges politisches Konzept zu verwirklichen. Georg Forster ist das Paradigma dieses Versuchs, die Republik in Deutschland einzuführen und auf der Herrschaft des Volkes begründete Verhältnisse zu schaffen. In einer Rede Über das Verhältnis der Mainzer gegen die Franken vor der Gesellschaft der Volksfreunde am 15. November 1792 betont er:

»Mitbürger! ... Ich finde mich in mein Gewissen gedrungen, öffentlich zu bekennen:

1. Daß mir die freieste Verfassung die beste scheint.

2. Daß wir es vor Gott und der Welt nicht verantworten könnten, wenn wir die Gelegenheit, wo wir eine Verfassung bekommen können, von uns stießen.

3. Daß man jedesmal, sooft es auf das dauerhafte Glück einer ganzen Stadt und eines Landes ankommt, auf einzelne Personen keine Rücksicht nehmen, viel weniger die Befriedigung einiger wenigen, wenn sie auch sonst unbescholten wären, die Freiheit und die damit verbundene moralische Veredlung aller opfern darf.

Endlich 4. Daß dies der glückliche, erwünschte Zeitpunkt einer wirklich ist, wo wir alle Kräfte anspannen müssen, um die Freiheit und Gleichheit, die unsere fränkischen Brüder uns darbieten, mit Eifer und warmen Dankgefühlen anzunehmen und mit Mut bis in den Tod für ihre Beibehaltung zu streiten. «**

* Claus Träger (Hg.), Mainz zwischen Rot und Schwarz. Die Mainzer Revolution 1792-1793 in Schriften, Reden und Briefen. Berlin 1963, S. 227.

* * Hierzu war ein Beitrag Bernd Weyergrafs vorgesehen, der aus Zeit- und Raumgründen (wie die übrigen angekündigten Beiträge) nicht erscheinen kann, aber einer gesonderten Publikation vorbehalten bleibt. Da dieser Beitrag unmittelbar zum Konzept dieses Bandes gehörte, seien hier kurz seine einzelnen Schritte dargestellt:

1. Darstellung der Position, die von Vertretern der literarischen Intelligenz zur Zeit des Vormärz (Gervinus u.a.), der nachrevolutionären Literaturgeschichtsschreibung und 
Dies Zitat, um sichtbar zu machen, wie sehr die politische Rede, statt Handlungsanweisung zu sein, unter Anrechnung dessen, was ihr realgeschichtlich widerfährt, zur monumentalen Geste geraten kann, allerdings einer Geste, auf die in diesem Fall zu achten wäre.

Gewiß wäre es wünschenswert gewesen, den vorliegenden Band "Deutsches Bürgertum und literarische Intelligenz $1750-1800$ « in folgenden Hinsichten durch ausgearbeitete Einzelbeiträge zu ergänzen:

Biographik zum späten 18. Jahrhundert (z.B. Wenck) und der 20er und 30er Jahre (u.a. Stern, Gerth, Balet) eingenommen wurde. Dann sollte die Darstellung und Kritik einschlägiger Arbeiten aus Bundesrepublik und DDR folgen: Enge Verknüpfung des sozialgeschichtlichen und biographischen Forschungsinteresses mit Fragen der Parteilichkeit, der Vermittlung und Aktualisierbarkeit (Probleme des "kulturellen Erbes" und des Weiterwirkens des "demokratischen und sozialistischen Vermächtnisses «). Analyse und Kritik von Positionen der BRD und Darstellung des ideologisch und wissenschaftspolitisch begründeten Desinteresses an der deutschen Spätaufklärung bis in die sechziger Jahre.

2. Skizze des historischen, ökonomischen und politischen Horizonts des späten 18. Jahrhunderts. Untersuchung der "Krise des Feudalismus " unter dem Gesichtspunkt, wie weit sie im Diskussionsprozeß, mit dem sich die literarische Intelligenz über ihre besondere Situation verständigt, als solche erkannt und dargestellt wird und somit zur Herausbildung eines Klassenbewußtseins beitragen kann. Ebenso Berücksichtigung der Bestrebungen, die dazu beitragen sollen, die sich im Schoß der feudalen Gesellschaftsordnung heranbildenden neuen Produktivkräfte durchzusetzen: Kritik des Merkantilismus, Propagierung physiokratischer Ideen, Diskussion agrarischer Produktionsformen, Kritik der Leibeigenschaft, pädagogische Reformen, Kritik der Arbeitsteilung usw. Da die so formulierten Bedürfnisse klassenspezifisch im Sinn der bürgerlichen Fraktionen (Handels- und Industriebürgertum) sind, liefern sie zugleich Indizien für die Klassenposition der einzelnen Autoren. Ebenso sollten hier die Bestrebungen unabhängiger Existenzsicherung, wie z. B. die Versuche eigenständiger landwirtschaftlicher Produktion (Merck, Wieland, Ziegenhagen u. a.), verlegerische Tätigkeit (Göschen, Perthes, Campe) erörtert werden.

3. Abgrenzung der verschiedenen Schichten des Bürgertums und der sie vertretenden Intelligenz, "abhängige " und "freie " Berufe; Vertreter des Bürgertums, die auf die "Treibhausatmosphäre " der territorialstaatlichen Wirtschaftspolitik oder auf die Nähe zum Hof angewiesen waren, und jene, denen an der Beseitigung der feudalen Produktionsverhältnisse insgesamt gelegen war.

4. Darstellung unterschiedlicher Formen der Ideologieproduktion; wirtschaftliche und politische Voraussetzungen für die Herausbildung eines bürgerlichen Klassenbewußtseins. Erfahrungshorizont der literarischen Intelligenz und Erwartungen in die Konsolidierung der eigenen Klasse. Erläuternd dazu: Klassenverständnis und Klassenidentität; Ideologie als Reflex auf die individuelle und gesellschaftliche Lage; Dialektik von Eigentum und Freiheit; Gleichheitsforderungen und Apologie der Ungleichheit; Verhältnis der literarischen Intelligenz zu den "niederen Volksklassen «; die Abgrenzung zu Klerus und Adel und zum vierten Stand; die Theorie der Klassenversöhnung.

5. Exemplarische Biographien, von denen aus das Problem der Wechselbeziehung von Klassenlage und politischer Haltung erörtert wird.

6. Darstellung der spezifischen Reaktionsweise einiger Vertreter der literarischen Intelligenz auf die Französische Revolution. Dabei stehen klassenbedingte bzw. schichtenspezifische Haltungen im Vordergrund. 
- Vergleich der politisch-sozialen Konstellationen in Frankreich und Deutschland vor und während der Französischen Revolution;

- Vergleich der beiden nationalen Entwicklungen bis zur Juli-Revolution;

- Daran anknüpfend, Vergleich der unterschiedlichen Auffassungen und Möglichkeiten des Schriftstellers in Frankreich und Deutschland;

- Analyse der romantischen Protestbewegung in Deutschländ, für die allerdings ein eigener Band von Literaturwissenschaft und Sozialwissenschaften in Vorbereitung ist;

- Analyse der Rezeptionsbedingungen der Französischen Revolution in Deutschland;

- Diskussion und Kritik des Begriffs "nationales Erbe«;

- deutsches Theater des 18. Jahrhunderts als Medium aufklärerischer Kritik und als bevorzugter Ort antifeudalistischer Propaganda;

- Klassische deutsche Philosophie und Ästhetik als Spiegel des neuen bürgerlichen Selbstverständnisses.

Ein solches Programm freilich würde weitere - umfangreiche - Bände erfordern. Überdies haben die Autoren dieses Bandes die Hoffnung, alle die angedeuteten Ergänzungspunkte zumindest implizit zur Genüge berücksichtigt zu haben.

Im Juni 1974

Bernd Lutz 